\title{
Optimal designs for estimating critical effective dose under model uncertainty in a dose response study
}

\author{
Holger Dette* ${ }^{*}$ Andrey Pepelyshev, Piter Shpilev and Weng Kee Wong ${ }^{\dagger \ddagger}$
}

Toxicologists have been increasingly using a class of models to describe a continuous response in the last few years. This class consists of nested nonlinear models and is used for estimating various parameters in the models or some meaningful function of the model parameters. Our work here is first to address design issues for this popular class of models among toxicologists. Specifically we construct a variety of optimal designs under model uncertainty and study their properties for estimating the critical effective dose (CED), which is model dependent. Two types of optimal designs are proposed: one type maximizes the minimum of efficiencies for estimating the CED regardless which member in the class of models is the appropriate model, and (ii) maximin compound optimal design that simultaneously selects the most appropriate model and provides the best estimate for CED at the same time. We compare relative efficiencies of these optimal designs and commonly used designs for estimating CED. To facilitate use of these designs, we have constructed a website that practitioners can generate tailor-made designs for their settings.

AMS 2000 SUBJECT CLASSIFICATIONS: 62K05.

KEYWORDS AND PHRASES: compound optimal design, critical effect size, local optimal design, maximin optimal design, model discrimination, robust design.

\section{INTRODUCTION}

This paper addresses design issues for dose response studies in toxicology when the main outcome is continuous and it is not known a priori which model is an appropriate one to use. Under this situation, one may consider a class of plausible models within which we believe lies an adequate model for fitting the data at hand. The statistical issues are how

*The work of Holger Dette was partially supported by the Sonderforschungsbereich 475, Komplexitätsreduktion in multivariaten Datenstrukturen (Teilprojekt A2) and in part by a NIH grant award R01GM072876 and a BMBF-grant SKAVOE.

†The work of Weng Kee Wong was partially by NIH grant awards R01GM072876, R24MD001762, P01 CA109091 and P30 CA16042-33.

${ }^{\ddagger}$ Corresponding author. to design the study to select the 'best' model from the class of models and at the same time able to estimate the critical effective dose (CED) in the selected model efficiently. The estimated CED is the dose that toxicologists use to estimate the dose that will result in a user-specified change in the continuous outcome after accounting for background noise. The user-specified change in the continuous outcome is usually expressed in terms of the critical effective size (CES) which is also pre-selected.

Ideally, we want the design to be able to identify the correct model from the postulated class of models and also provide an efficient estimate for the CED, which is a function of the parameters in the identified model. In this paper, it is further assumed for simplicity that there is only one independent variable, the dose level. The design space is the range of dose levels of interest where the researcher selects the dose levels to observe the outcome. Throughout, we assume all design issues have to be decided in advance of the study and so sequential designs are not considered.

The researcher has to select the number of dose levels from the design space to observe the continuous outcome, decide where these dose levels are and the number of replicates at each of these dose levels. We further generalize the design problem to one for finding continuous designs, meaning that we now view designs as probability measures on the design space. Continuous designs were proposed by Kiefer in the late 1950s and have been shown to be much more amendable to analytical description and study than exact designs. Throughout we denote a continuous design with mass $p_{i}$ at $x_{i}, i=1, \ldots, k$ by $\xi=\left\{x_{1}, x_{2}, \ldots, x_{k} ; p_{1}, p_{2}, \ldots, p_{k}\right\}$.

Here is a simple illustration of a continuous design on the design space $[0,25]$. Let $\xi$ be a continuous design that takes half the observations at dose level 5 and half at dose level 20 . We denote this design by writing $\xi=\{5,20 ; 1 / 2,1 / 2\}$; the first part denotes the two dose levels and the latter part denotes the corresponding proportion of observations to be taken at each of the dose levels. An optimal (continuous) design is one that maximizes or minimizes a given optimality criterion over all designs on the design space. Further details and motivations for working with continuous designs are given in the voluminous collection of papers by Kiefer and edited by Brown et al. (1985). Optimal rounding proce- 
dures to convert a continuous design to an exact design for implementation are given in Rieder and Pukelsheim (1992).

Addressing design issues invariably requires model assumptions that specify how the mean outcome relates to the independent variable. Usually a specific functional form is assumed either from experts' opinions or from the science of the problem, see Gaylor and Chen (1993), Catalano et al. (1993), Slob and Pieters (1998), Oscar (2004), Moerbeek, Piersma and Slob (2004), among many others. When it is problematic to specify a single model to describe the functional relationship between the mean outcome and the dose level, a common strategy is to work with a class of plausible nested models assumed to include the 'true' model. This class of models is usually arrived at after consultation with experts in the area. The plausible models within this class should be nested allowing one to be built upon another; typically this means the 'largest' model has the largest number of parameters and the next 'largest' model is obtained from the 'largest' one by specifying one or more parameters equal to some user-selected fixed values. Dette, Melas and Wong (2005) considered a simple class of plausible models consisting of either the Emax and Michaelis-Menten models. These are very popular models in the biological sciences with the Emax model defined by

$$
E(Y)=\frac{a x^{h}}{b+x^{h}} .
$$

Here $Y$ typically is the velocity of the enzyme kinetics and $x$ is the substrate concentration. When $h=1$, the Emax model reduces to the Michaelis-Menten. Other values of $h$ permit greater flexibility for the Emax model to capture skewed and steep responses as the substrate concentration varies. Once this class is identified, one works assuming the 'largest' model holds. For example, Dette, Melas and Wong (2005) sought an efficient design to estimate $h$ in the Emax model as accurately as possible and at the same time also have efficient estimates for the parameters $a$ and $b$ should the Michaelis-Menten model hold.

The motivation for this work comes from repeated proposals recently in the toxicology literature to use a class of models to study a continuous outcome in toxicological studies [Moerbeek, Piersma and Slob (2004), Piersma et al. (2002), Woutersen et al. (2001), Slob (2002)]. In all these papers, the interest was only in estimation problems and so they did not consider design issues. As is typical in such publications, the rationale for their designs is not explained. Here are a few examples of designs used in toxicological studies and their outcomes. In Piersma et al. (2002), rats were prenatally exposed to diethylstilbestrol and the design had 16 animals in each of the 10 dose groups at $0,1.0,1.7$, $2.8,4.7,7.8,13,22,36$ and $60 \mathrm{mg} / \mathrm{kg}$ body weight per day. In Woutersen et al. (2001), rats were exposed to Rhodorsil Silane in a 28-day toxicity study and 3 designs were employed: the first one had 10 rats in each of the 7 dose groups $(7 \times 10$ design $)$ and the second had 5 rats in each of the 7 dose groups; the 7 dose groups were $0,50,150,300,450,600$ and $750 \mathrm{mg} / \mathrm{kg}$ body weight/day. The third had $10 \mathrm{rats}$ in each of the 4 dose groups at 0, 50, 150 and $450 \mathrm{mg}$ Rhodorsil Silane/kg body weight/day. There were many continuous outcomes in each of these studies. The continuous outcomes in these studies ranged from maternal body weight on gestation day 21, maternal serum estradiol concentration at gestation day 21, weights of fetuses at gestation day 21 , measures in haematology and clinical chemistry to immunological responses such as IgG and IgM to sheep red blood cell challenge and pup weights at days 1 and 21 . In Woutersen et al. (2001), their main goals were to estimate various critical effective doses; these are doses that will result in a user-specified level of toxicity found in rats over the background noise. In the discussion section, we comment on the performance of these designs.

In this paper, we develop optimal designs for identifying an appropriate model within the class of models and also at the same time provide a reliable estimate for the critical effect dose (CED) in the selected model. Design issues are always difficult to address and we begin first by considering local optimal designs because they are the easiest to construct for nonlinear models (Chernoff, 1953). These designs require the user to supply nominal values of the model parameters before the optimal design can be constructed. Nominal values represent the best guess for the true values of the set of parameters and are usually obtained either from prior similar experiments or experts' opinions. When model assumptions are mis-specified, local optimal design can lose substantial efficiency. To overcome this risk, we propose maximin optimal designs that have been shown to be robust to mis-specification of model assumptions in other settings, see for example, Biedermann, Dette and Pepelyshev (2006) and Dette et al. (2008). These maximin optimal designs maximize the minimum efficiency regardless which model in the class of models is the appropriate model. As such, these optimal designs provide some protection against picking a wrong model from the postulated class. We also construct a compound optimal design to account for the dual objectives of discriminating models and estimating the CED simultaneously.

In Section 2, we describe the class of nonlinear models and the design criterion for estimating CED. We describe relationships among models in the class and provide local optimal design for estimating the CED for each member in the class. We also show how an optimal design constructed for a specific setup can be used to deduce the optimal design under another setup where assumptions on the design space and model parameters are different. In Section 3, we construct maximin optimal designs and compound optimal designs for toxicology studies and assess their robustness properties to model mis-specification and their effectiveness for discriminating between models and estimating CED at the same time. We also compare performance of selected uniform designs that are intuitively appealing to practitioners. 
These designs take an equal number of observations over a set of equally spaced dose levels. In Section 4, we discuss four practical issues. First, we construct and compare corresponding optimal designs for log-normally distributed outcomes, which is another popular assumption used by toxicologists. Secondly, we investigate efficiencies of several designs used by toxicologists. Thirdly, we perform a simulation study to assess how our optimal designs perform in a real example with a relatively small sample size. Finally, we introduce the reader to our design web site that the reader can use freely to generate a variety of optimal designs for a broad range of models frequently used in the biological sciences, including optimal designs proposed in this paper. We conclude the paper by noting that our proposed design strategy represents a first step in finding an efficient design for this difficult problem. For most practical applications, optimal designs found here will have to be refined sequentially before they can be implemented.

\section{MODEL UNCERTAINTY AND CED-OPTIMALITY}

Moerbeek, Piersma and Slob (2004), Woutersen et al. (2001), Piersma et al. (2002) and Slob (2002) used the following class of models in several toxicological studies. These authors showed with justifications that the class of models is sufficiently flexible to accommodate typical continuous outcomes of interest in toxicological studies. For each of these models defined on the given design space $[0, T], Y$ is the response and $t$ is the dose level:

$$
\begin{aligned}
& E(Y)=a e^{-b t} \text { with } a>0, b>0 \\
& E(Y)=a e^{-b t^{d}} \text { with } a>0, b>0, d \geq 1 \\
& E(Y)=a\left(c-(c-1) e^{-b t}\right) \text { with } a>0, b>0, c \geq 0 \\
& E(Y)=a\left(c-(c-1) e^{-b t^{d}}\right) \\
& \quad \text { with } a>0, b>0, c \geq 0, d \geq 1 .
\end{aligned}
$$

This class consists of models nested within one another, where 'smaller' models can be obtained from the 'largest' model by setting specific parameters in the 'largest' model equal to specific values. All observations are assumed to be independent normally distributed with the same variance, say $\sigma^{2}>0$, and the expectation of $Y$ observed at $t$ is given by

$$
E[Y]=\eta(t, \theta)
$$

where $\eta(t, \theta)$ is one of the functions (2.3)-(2.6) and all parameters in the mean response are components of the $p \times 1$ vector parameter $\theta$. In what is to follow, we suppress the $p$ dimensional parameter $\theta$ in $\eta(t, \theta)$ for simplicity when there is no confusion.

In toxicological studies with a continuous outcome, the benchmark response is usually expressed in terms of a critical effect size (CES). This is the amount that we expect the percent change in the average level of the outcome compared with the background noise. In practice, CES is user-specified and traditionally set equal to 0.05 or 0.10 . For a given mean response $\eta(t)$ and a user-selected CES, the critical effective dose CED is calculated from

$$
\mathrm{CES}=\frac{\eta(0)-\eta(\mathrm{CED})}{\eta(0)}
$$

if $\eta(t)$ is a decreasing function. All our functions $\eta(t)$ defined in (2.3)-(2.6) are decreasing.

The parameters in the above models may or may not all have meaningful interpretations, but frequently a reparametrization of the mean function or working with a function of the model parameters has a practical meaning. By inverting the above functions, such as the mean function in (2.6), it is straightforward to show that

$$
\mathrm{CED}=\operatorname{CED}(b, d, c)=\left(-\frac{\ln \frac{c-1+\mathrm{CES}}{c-1}}{b}\right)^{\frac{1}{d}} .
$$

The corresponding expressions for CED for other models can be directly deduced by setting $c=0$ for models (2.3) and (2.4) and by setting $d=1$ for models (2.3) and (2.5). Thus the CED is the dose that results in a percent change in the mean response relative to the background noise and the magnitude of the anticipated change is specified by the critical effect size.

To estimate the confidence interval for CED for a specific model using design $\xi$, one uses the delta method to obtain its asymptotic variance and then finds a design to minimize it. Specifically, we have

$$
\operatorname{Var}(\widehat{\mathrm{CED}}) \approx \frac{\sigma^{2}}{N} \Phi(\xi)
$$

where

$$
\Phi(\xi)=g^{T}(\theta) M^{-}(\xi, \theta) g(\theta) \quad \text { and } \quad g(\theta)=\frac{\partial \mathrm{CED}}{\partial \theta} .
$$

For the vector of the parameters $\theta=(a, b, d, c)^{T}$ the local CED-optimal design minimizes the function $\Phi(\xi)$ by choice of the design $\xi$. The matrix $M(\xi, \theta)$ in the above expression is the information matrix for the specific model $\eta(t)$ using an arbitrary design $\xi$ and $M^{-}(\xi, \theta)$ is a generalized inverse of $M(\xi, \theta)$. We call a design nonsingular if its information is nonsingular; otherwise it is a singular design. For a specific model $\eta$, let $f(t, \theta)=\frac{\partial \eta(t, \theta)}{\partial \theta}$ and recall that the information matrix is given by

$$
M(\xi, \theta)=\int_{0}^{T} f(t, \theta) f^{T}(t, \theta) d \xi(t) .
$$

The corresponding regression vectors $f(t, \theta)$ for different 
models are

$$
\begin{aligned}
f^{(2.3)}(t, \theta)= & f(t, a, b)=\left(e^{-b t},-a t e^{-b t}\right)^{T}, \\
f^{(2.4)}(t, \theta)= & f(t, a, b, d) \\
= & \left(e^{-b t^{d}},-a t^{d} e^{-b t^{d}},-a b t^{d} \ln (t) e^{-b t^{d}}\right)^{T}, \\
f^{(2.5)}(t, \theta)= & \left(c-(c-1) e^{-t b}, a(c-1) t e^{-t b}, a\left(1-e^{-t b}\right)\right)^{T}, \\
f^{(2.6)}(t, \theta)= & \left(c-(c-1) e^{-b t^{d}}, a(c-1) t^{d} e^{-b t^{d}},\right. \\
& \left.a(c-1) t^{d} \ln (t) b e^{-b t^{d}}, a\left(1-e^{-b t^{d}}\right)\right)^{T}
\end{aligned}
$$

and the corresponding vectors $g(\theta)$ are

$$
\begin{aligned}
& g^{(2.3)}(\theta)=g(a, b)=\left(0, \frac{\ln (1-\mathrm{CES})}{b^{2}}\right)^{T}, \\
& g^{(2.4)}(\theta)=\left(0,-\frac{1}{d b}\left(-\frac{\ln (1-\mathrm{CES})}{b}\right)^{\frac{1}{d}}\right. \\
& \left.-\frac{1}{d^{2}}\left(-\frac{\ln (1-\mathrm{CES})}{b}\right)^{\frac{1}{d}} \ln \left(-\frac{\ln (1-\mathrm{CES})}{b}\right)\right)^{T}, \\
& g^{(2.5)}(\theta)=\left(0, \frac{\ln \frac{c-1+\mathrm{CES}}{c-1}}{b^{2}}, \frac{1-\frac{c-1+\mathrm{CES}}{c-1}}{b(c-1+\mathrm{CES})}\right)^{T} \text {, } \\
& g^{(2.6)}(\theta)=\left(0,-\frac{1}{d b}\left(-\frac{\ln \frac{c-1+\mathrm{CES}}{c-1}}{b}\right)^{\frac{1}{d}}\right. \\
& -\frac{1}{d^{2}}\left(-\frac{\ln \frac{c-1+\mathrm{CES}}{c-1}}{b}\right)^{\frac{1}{d}} \ln \left(-\frac{\ln \frac{c-1+\mathrm{CES}}{c-1}}{b}\right), \\
& \left.\left(-\frac{\ln \frac{c-1+\mathrm{CES}}{c-1}}{b}\right)^{\frac{1}{d}} \frac{1-\frac{c-1+\mathrm{CES}}{c-1}}{d(c-1+\mathrm{CES}) \ln \frac{c-1+\mathrm{CES}}{c-1}}\right)^{T} \text {. }
\end{aligned}
$$

The next five technical results provide analytical descriptions and properties of local CED-optimal design for each model. The first one shows that local CED-optimal design does not depend on the value of the parameter $a$, and the next four results describe the structure of the local CEDoptimal designs for the four nonlinear models (2.3)-(2.6). We provide an illustrative proof of our results for model (2.3) only; the arguments for the other models are similar. For our class of models, the results also show how optimal design for a particular design setting can be deduced from another design setting by only considering values of $b$ and $T$. Our technical justifications use a celebrated geometric result called Elfving's theorem which is widely discussed in design monographs, such as in Pázman (1986, p. 71) or Pukelsheim (1993, p. 50). We provide only proofs for Lemma 2.1 and 2.2; the rest are similar. All support points of the optimal design are denoted by $t_{i}^{*}$ and its weight by $w_{i}^{*}$; clearly these support points and the weights depend on the model parameters and the lemmas also show how they vary as model parameters take on different values. These relationships allow us to deduce optimal design for one set of model parameters from another.

Lemma 2.1. A local CED-optimal design does not depend on $a$.
Proof. The statement follows from the fact that an optimality function have a form

$$
\Phi(\xi, \theta)=a^{2-2 p} \Phi(\xi, \tilde{\theta})
$$

where $\left(a, \tilde{\theta}^{T}\right)=\theta^{T} \in \mathbb{R}^{p}$ and $p$ is the number of parameters.

Lemma 2.2. Let $u^{*} \approx 1.278$ be a unique solution of equation $e^{-u}=u-1$. For model (2.3) a local CED-optimal design does not depend on a and CES and is given by

$$
\left\{0, u^{*} / b ; \frac{e^{-u^{*}}}{1+e^{-u^{*}}}, \frac{1}{1+e^{-u^{*}}}\right\}
$$

if $T>u^{*} / b$; otherwise it is given by

$$
\left\{0, T ; \frac{e^{-b T}}{1+e^{-b T}}, \frac{1}{1+e^{-b T}}\right\} .
$$

Proof. By Elfving's theorem, there exists a representation

$$
v g=w_{1}^{*} f\left(t_{1}^{*}\right)-w_{2}^{*} f\left(t_{2}^{*}\right)
$$

for some $v \in \mathbb{R}$ and $g^{T} M^{-}\left(\xi^{*}\right) g=1 / v^{2}$. Moreover, points of optimal design lie on the boundary of Elfving set. Thus, one of the support points of the optimal design is $t_{1}^{*}=0$. For large enough values of $T$, the point $f\left(t_{2}\right)$ belongs to the boundary if $t_{2}$ is small; otherwise it does not. The crucial value of $t_{2}$ is a solution of the equation

$$
\frac{f_{2}^{\prime}\left(t_{2}\right)}{f_{1}^{\prime}\left(t_{2}\right)}=\frac{f_{2}\left(t_{2}\right)+f_{2}(0)}{f_{1}\left(t_{2}\right)+f_{1}(0)}
$$

Straightforward calculation shows the other support point of the optimal design is at $t_{2}^{*}=u^{*} / b$. From the equation for the first coordinate of (2.7), we determine directly the weights $w_{1}^{*}$ and $w_{2}^{*}$ for the optimal design.

Lemma 2.3. For model (2.4), the local CED-optimal design has one of three possible forms. It is either given by a 2-point singular design

$$
\left\{0, C E D ; \frac{1-C E S}{2-C E S}, \frac{1}{2-C E S}\right\}
$$

if the parameter $b$ is small enough, or it has the form

$$
\left\{0, t_{2}^{*}, t_{3}^{*} ; w_{1}^{*}, w_{2}^{*}, w_{3}^{*}\right\}
$$

if $t_{3}^{*}<T$; otherwise the optimal design takes on the form

$$
\left\{0, t_{2}^{*}, T ; w_{1}^{*}, w_{2}^{*}, w_{3}^{*}\right\} .
$$

Moreover, for 3-point optimal designs, we have

$$
\begin{array}{rlrl}
t_{i}^{*}(b, d, T) & =T t_{i}^{*}\left(b T^{d}, d, 1\right), & w_{i}^{*}(b, d, T)=w_{i}^{*}\left(b T^{d}, d, 1\right) . \\
t_{i}^{*}(b, 1,1)=\left(t_{i}^{*}(b, d, 1)\right)^{d}, & w_{i}^{*}(b, 1,1)=w_{i}^{*}(b, d, 1) .
\end{array}
$$


Table 1. Local CED-optimal designs for model (2.3) and for model (2.6) with $d=1, c=0$ on the design space $[0, T]$ for various values of the parameter $b$ and $\mathrm{CES}=0.05$

\begin{tabular}{|c|c|c|c|c|c|c|c|c|c|c|c|c|c|}
\hline \multirow[b]{2}{*}{$T$} & \multirow[b]{2}{*}{$b$} & \multicolumn{4}{|c|}{ model (2.3) } & \multicolumn{8}{|c|}{ model $(2.6)$} \\
\hline & & $t_{1}$ & $t_{2}$ & $w_{1}$ & $w_{2}$ & $t_{1}$ & $t_{2}$ & $t_{3}$ & $t_{4}$ & $w_{1}$ & $w_{2}$ & $w_{3}$ & $w_{4}$ \\
\hline 1 & 0.5 & 0 & 1 & 0.377 & 0.623 & 0 & 0.103 & & & 0.487 & 0.513 & & \\
\hline 5 & 0.1 & 0 & 5 & 0.377 & 0.623 & 0 & 0.513 & & & 0.487 & 0.513 & & \\
\hline 5 & 0.5 & 0 & 2.557 & 0.218 & 0.782 & 0 & 0.430 & 2.482 & 5 & 0.337 & 0.463 & 0.141 & 0.058 \\
\hline 5 & 1.0 & 0 & 1.278 & 0.218 & 0.782 & 0 & 0.277 & 1.718 & 5 & 0.316 & 0.454 & 0.159 & 0.071 \\
\hline
\end{tabular}

Table 2. Local CED-optimal designs for model (2.4) with $d=1$ and for model (2.5) with $c=0$ on the design space $[0, T]$ for various values of the parameter $b$ and $\mathrm{CES}=0.05$

\begin{tabular}{|c|c|c|c|c|c|c|c|c|c|c|c|c|c|}
\hline \multirow[b]{2}{*}{$T$} & \multirow[b]{2}{*}{$b$} & \multicolumn{6}{|c|}{ model (2.4) } & \multicolumn{6}{|c|}{ model (2.5) } \\
\hline & & $t_{1}$ & $t_{2}$ & $t_{3}$ & $w_{1}$ & $\overline{w_{2}}$ & $w_{3}$ & $t_{1}$ & $t_{2}$ & $t_{3}$ & $w_{1}$ & $\overline{w_{2}}$ & $w_{3}$ \\
\hline 1 & 0.1 & 0 & 0.513 & & 0.487 & 0.513 & & 0 & 0.513 & & 0.487 & 0.513 & \\
\hline 1 & 0.5 & 0 & 0.305 & 1 & 0.378 & 0.515 & 0.106 & 0 & 0.459 & 1 & 0.353 & 0.532 & 0.115 \\
\hline 1 & 1.0 & 0 & 0.251 & 1 & 0.344 & 0.511 & 0.145 & 0 & 0.418 & 1 & 0.317 & 0.554 & 0.129 \\
\hline 5 & 0.1 & 0 & 1.523 & 5 & 0.378 & 0.515 & 0.106 & 0 & 2.293 & 5 & 0.353 & 0.532 & 0.115 \\
\hline 5 & 0.5 & 0 & 0.672 & 4.507 & 0.305 & 0.462 & 0.233 & 0 & 1.553 & 5 & 0.261 & 0.593 & 0.146 \\
\hline 5 & 1.0 & 0 & 0.336 & 2.253 & 0.305 & 0.462 & 0.233 & 0 & 0.966 & 5 & 0.232 & 0.613 & 0.155 \\
\hline
\end{tabular}

For a 3-point optimal design, Elfving theorem implies that the weights of the optimal design are solutions of the equation

$$
\left(g \vdots f\left(t_{3}^{*}\right)-f(0) \vdots f\left(t_{3}^{*}\right)+f\left(t_{2}\right)\right)\left(v, w_{1}, w_{2}\right)^{T}=f\left(t_{3}^{*}\right)
$$

and $t_{2}=t_{2}^{*}$ is the middle support point of the optimal design that solves $\partial v / \partial t_{2}=0$ where

$$
v=v\left(t_{2}\right)=\frac{\operatorname{det}\left(f\left(t_{3}^{*}\right) \vdots f\left(t_{3}^{*}\right)-f(0) \vdots f\left(t_{3}^{*}\right)+f\left(t_{2}\right)\right)}{\operatorname{det}\left(g \vdots f\left(t_{3}^{*}\right)-f(0) \vdots f\left(t_{3}^{*}\right)+f\left(t_{2}\right)\right)} .
$$

There is no explicit solution for model (2.4) but there is an explicit solution for model (2.5) as seen in the next lemma.

Lemma 2.4. For model (2.5), the local CED-optimal design has one of two forms. It is either given by a 2-point singular design

$$
\left\{0, C E D ; \frac{1-C E S}{2-C E S}, \frac{1}{2-C E S}\right\}
$$

if the parameter $b$ is small enough or $c$ is large enough; otherwise, it has the following form

$$
\left\{0, t_{2}^{*}, T ; w_{1}^{*}, w_{2}^{*}, w_{3}^{*}\right\}
$$

where

$$
t_{2}^{*}=t_{2}^{*}(b, c, T)=\frac{1-(1+b T) e^{-b T}}{b\left(1-e^{-b T}\right)} .
$$

Moreover, for 3-point optimal designs, we have

$$
t_{i}^{*}(b, c, T)=T t_{i}^{*}(b T, c, 1), \quad w_{i}^{*}(b, c, T)=w_{i}^{*}(b T, c, 1),
$$

and the points $t_{i}^{*}(b, c, T)$ do not depend on $c$.

Lemma 2.5. For model (2.6), the local CED-optimal design has one of two form. It is either a singular 2-point design given by

$$
\left\{0, C E D ; \frac{1-C E S}{2-C E S}, \frac{1}{2-C E S}\right\}
$$

if the parameter $b$ is small enough or $c$ is large enough; otherwise it has the form

$$
\left\{0, t_{2}^{*}, t_{3}^{*}, T ; w_{1}^{*}, w_{2}^{*}, w_{3}^{*}, w_{4}^{*}\right\} .
$$

Moreover, for 4-point optimal designs, we have

$t_{i}^{*}(b, d, c, T)=T t_{i}^{*}\left(b T^{d}, d, c, 1\right), \quad w_{i}^{*}(b, d, c, T)=w_{i}^{*}\left(b T^{d}, d, c, 1\right)$,

$t_{i}^{*}(b, 1, c, 1)=\left(t_{i}^{*}(b, d, c, 1)\right)^{d}, \quad w_{i}^{*}(b, 1, c, 1)=w_{i}^{*}(b, d, c, 1)$

and the points $t_{i}^{*}(b, d, c, T)$ do not depend on $c$.

Tables 1 and 2 show local CED-optimal designs for each of the four models when CES $=0.05$ and selected values for $b$ and $T$. As is described in the above results, the local CED-optimal design may be a singular 2-point design for models (2.4)-(2.6) or a saturated design where the number of points equal to the number of model parameters.

\section{MAXIMIN CED-OPTIMAL DESIGN AND COMPOUND OPTIMAL DESIGN}

The local optimal design for estimating CED depends on the assumed model and the nominal values of the model parameters. When the nominal values are mis-specified, local 
Table 3. Maximin CED-optimal designs for models (2.3)-(2.6) on the design space $[0, T]$ for various values of the parameter $b$ with $d=1, c=0$ and $\mathrm{CES}=0.05$ and their efficiencies

\begin{tabular}{cccccccccccccc}
\hline$T$ & $b$ & $t_{1}$ & $t_{2}$ & $t_{3}$ & $t_{4}$ & $w_{1}$ & $w_{2}$ & $w_{3}$ & $w_{4}$ & eff $_{\mathrm{CED}}^{(2.3)}$ & eff $_{\mathrm{CED}}^{(2.4)}$ & eff $_{\mathrm{CED}}^{(2.5)}$ & eff $_{\mathrm{CED}}^{(2.6)}$ \\
\hline 1 & 0.1 & 0 & & 0.513 & 1 & 0.417 & & 0.296 & 0.287 & 0.686 & 0.686 & 0.686 & 0.692 \\
1 & 0.5 & 0 & 0.183 & 0.612 & 1 & 0.299 & 0.228 & 0.231 & 0.242 & 0.597 & 0.636 & 0.597 & 0.597 \\
1 & 1.0 & 0 & 0.170 & 0.594 & 1 & 0.261 & 0.217 & 0.288 & 0.234 & 0.615 & 0.615 & 0.615 & 0.615 \\
5 & 0.1 & 0 & 0.914 & 3.059 & 5 & 0.299 & 0.228 & 0.231 & 0.242 & 0.597 & 0.636 & 0.597 & 0.597 \\
5 & 0.5 & 0 & 0.820 & 2.451 & 5 & 0.237 & 0.292 & 0.350 & 0.121 & 0.655 & 0.655 & 0.655 & 0.655 \\
5 & 1.0 & 0 & 0.529 & 1.545 & 5 & 0.236 & 0.366 & 0.326 & 0.072 & 0.639 & 0.639 & 0.639 & 0.673 \\
\hline
\end{tabular}

optimal designs can lose substantial efficiency. This problem is further compounded when there is model uncertainty. This implies that local optimal designs while potentially useful as a starting point, are unlikely to be adequate for practical implementation. However, local optimal designs are useful as a first step to constructing more versatile and robust designs to model assumptions. We now discuss two design strategies that utilize local optimal designs.

The maximin approach of designing a study provides an alternative that can be appealing [see Dette (1995), Müller (1995) and Müller and Pázman (1998) among others]. Procedurally, one first considers the efficiency of a design for estimating the CED relative to each of the models; among these relative efficiencies, the maximin CED-optimal design is the one that maximizes the minimum of these relative efficiencies. Technically, for a fixed $\theta$ we call the design that maximizes

$$
\min \left\{\operatorname{eff}_{\mathrm{CED}}^{(2.3)}(\xi, \theta), \operatorname{eff}_{\mathrm{CED}}^{(2.4)}(\xi, \theta), \operatorname{eff}_{\mathrm{CED}}^{(2.5)}(\xi, \theta), \operatorname{eff}_{\mathrm{CED}}^{(2.6)}(\xi, \theta)\right\}
$$

over all designs on the design space a maximin CEDoptimal design, where CED-efficiency (for each model) is given by

$$
\operatorname{eff}_{\mathrm{CED}}(\xi)=\frac{\min _{\tilde{\xi}} \Phi(\tilde{\xi})}{\Phi(\xi)}
$$

The last ratio is obviously a number between 0 and 1 and represents the reduction in sample size from use of the optimal design compared with using the design $\xi$ for the same level of precision for estimating CED. For example if $\operatorname{eff}_{\mathrm{CED}}(\xi)=0.5$, the design $\xi$ needs to be replicated twice to obtain an CED estimate as accurate as the estimate from the local optimal design. As expected, maximin optimal designs are difficult to find and defy analytical description. They have to be found numerically and several maximin optimal designs are shown in Table 3 for selected values of $b$ and $T$, along with their efficiencies relative to the local CEDoptimal designs for models (2.3)-(2.6).

In practice, maximin CED-optimal designs are found by first maximizing the optimality criterion within the class of all 4-point designs on the given design space. This is because 4 points are required for CED estimation in all models (2.3)(2.6). The optimization is performed with the NelderMead algorithm in the MATLAB package. After the optimal 4-point
Table 4. Maximin compound designs for models (2.3)-(2.6) on the design space $[0, T]$ for various values of the parameter $b$ with $d=1, c=0$ and $\mathrm{CES}=0.05$

\begin{tabular}{cccccccccc}
\hline$T$ & $b$ & $t_{1}$ & $t_{2}$ & $t_{3}$ & $t_{4}$ & $w_{1}$ & $w_{2}$ & $w_{3}$ & $w_{4}$ \\
\hline 1 & 0.1 & 0 & 0.173 & 0.622 & 1 & 0.361 & 0.152 & 0.281 & 0.205 \\
1 & 0.5 & 0 & 0.183 & 0.612 & 1 & 0.299 & 0.228 & 0.231 & 0.242 \\
1 & 1.0 & 0 & 0.170 & 0.594 & 1 & 0.261 & 0.217 & 0.288 & 0.234 \\
5 & 0.1 & 0 & 0.914 & 3.059 & 5 & 0.299 & 0.228 & 0.231 & 0.242 \\
5 & 0.5 & 0 & 0.698 & 2.398 & 5 & 0.186 & 0.225 & 0.298 & 0.291 \\
5 & 1.0 & 0 & 0.639 & 1.899 & 5 & 0.147 & 0.267 & 0.240 & 0.347 \\
\hline
\end{tabular}

design is found, we next search for the optimal design within the class of all 5 points designs, and repeat the procedure, each time increasing the number of points by unity, until no reduction in the criterion value is observed.

We can further extend our design strategy to discriminate models and estimating parameters at the same time using a maximin approach. To do this, we first set the design criteria for discriminating between each pair of models and for CED estimation. Specifically, for a fixed $\theta$ we call the design that maximizes

$$
\begin{array}{r}
\min \left\{\operatorname{eff}_{\mathrm{CED}}^{(2.3)}(\xi, \theta), \mathrm{eff}_{\mathrm{CED}}^{(2.4)}(\xi, \theta), \mathrm{eff}_{\mathrm{CED}}^{(2.5)}(\xi, \theta),\right. \\
\operatorname{eff}_{\mathrm{CED}}^{(2.6)}(\xi, \theta), \mathrm{eff}^{(2.4)-(2.3)}(\xi, \theta), \mathrm{eff}^{(2.5)-(2.3)}(\xi, \theta), \\
\left.\operatorname{eff}^{(2.6)-(2.5)}(\xi, \theta), \mathrm{eff}^{(2.6)-(2.4)}(\xi, \theta)\right\}
\end{array}
$$

over all designs on the design space a maximin compound design where eff ${ }^{\left(M_{1}\right)-\left(M_{2}\right)}(\xi, \theta)$ is an efficiency of design $\xi$ for discrimination two models $M_{1}$ and $M_{2}$. Such designs are efficient for CED estimation in the selected model after model discrimination. Table 4 displays exemplary maximin optimal designs and Table 5 shows their efficiencies for estimating CED under each model and for discriminating between pairs of models.

Our proposed maximin compound designs are still local in the sense that they depend on nominal values of the parameters. One could in principle incorporate the uncertainty of the nominal values at the design stage as well similar to the approach proposed in Biedermann, Dette and Pepelyshev (2006), Dette et al. (2008). We do note that our small scale investigation showed that maximin optimal designs are not sensitive to small changes in the nominal values of the parameters in the models considered here. 
Table 5. CED-Efficiencies and efficiencies of discrimination of maximin compound designs for models (2.3)-(2.6) on the design space $[0, T]$ for various values of the parameter $b$ with $d=1, c=0$ and $\mathrm{CES}=0.05$

\begin{tabular}{|c|c|c|c|c|c|c|c|c|c|}
\hline \multirow[b]{2}{*}{$T$} & \multirow[b]{2}{*}{$b$} & \multicolumn{4}{|c|}{ CED efficiency } & \multicolumn{4}{|c|}{ Efficiency of discrimination } \\
\hline & & $(2.3)$ & $(2.4)$ & $(2.5)$ & $(2.6)$ & $(2.4)-(2.3)$ & $(2.5)-(2.3)$ & $(2.6)-(2.5)$ & $(2.6)-(2.4)$ \\
\hline 1 & 0.1 & 0.610 & 0.620 & 0.620 & 0.610 & 0.622 & 0.651 & 0.610 & 0.694 \\
\hline 1 & 1.0 & 0.615 & 0.615 & 0.615 & 0.615 & 0.643 & 0.626 & 0.743 & 0.803 \\
\hline 5 & 0.1 & 0.597 & 0.637 & 0.597 & 0.597 & 0.677 & 0.634 & 0.720 & 0.737 \\
\hline 5 & 0.5 & 0.605 & 0.633 & 0.605 & 0.605 & 0.697 & 0.605 & 0.716 & 0.813 \\
\hline
\end{tabular}

Table 6. Maximin CED-optimal designs for models (2.3)-(2.6) with lognormality assumption on the design space $[0, T]$ for various values of the parameter $b$ with $d=1, c=0$ and $\mathrm{CES}=0.05$ and their efficiencies

\begin{tabular}{|c|c|c|c|c|c|c|c|c|c|c|c|c|c|}
\hline$T$ & $b$ & $t_{1}$ & $t_{2}$ & $t_{3}$ & $t_{4}$ & $w_{1}$ & $w_{2}$ & $w_{3}$ & $w_{4}$ & $\mathrm{eff}_{\mathrm{CED}}^{(2.3)}$ & $\mathrm{eff}_{\mathrm{CED}}^{(2.4)}$ & $\mathrm{eff}_{\mathrm{CED}}^{(2.5)}$ & $e_{\mathrm{CED}}^{(2.6)}$ \\
\hline 1 & 0.1 & 0 & & 0.513 & 1 & 0.430 & & 0.289 & 0.281 & 0.691 & 0.691 & 0.691 & 0.691 \\
\hline 1 & 0.5 & 0 & 0.196 & 0.638 & 1 & 0.334 & 0.224 & 0.221 & 0.221 & 0.620 & 0.655 & 0.620 & 0.620 \\
\hline 1 & 1.0 & 0 & 0.195 & 0.655 & 1 & 0.326 & 0.202 & 0.240 & 0.232 & 0.636 & 0.636 & 0.636 & 0.636 \\
\hline 5 & 0.1 & 0 & 0.982 & 3.191 & 5 & 0.334 & 0.224 & 0.221 & 0.221 & 0.620 & 0.655 & 0.620 & 0.620 \\
\hline 5 & 0.5 & 0 & 1.188 & 3.485 & 5 & 0.334 & 0.198 & 0.238 & 0.231 & 0.643 & 0.643 & 0.643 & 0.643 \\
\hline 5 & 1.0 & 0 & 1.450 & 3.758 & 5 & 0.347 & 0.204 & 0.239 & 0.211 & 0.644 & 0.644 & 0.644 & 0.644 \\
\hline
\end{tabular}

\section{DISCUSSION}

This closing section has four purposes aimed at the practitioners. The first purpose is to address distributional assumption on the error terms; in particular we construct optimal designs under the assumption of log-normality and compare results obtained under the normality assumption. Secondly, we discuss efficiencies of designs used by toxicologists relative to our proposed optimal designs. The third purpose is to evaluate how well our maximin optimal designs perform in practice using a small simulation study. The final purpose is to draw attention to our design web site where many types of optimal designs for several models can be generated under user-specified settings. We end the paper with closing remarks on how to implement the proposed optimal designs in practice.

\subsection{Distributional assumptions}

Sometimes toxicologists prefer to assume the continuous outcomes are log-normally distributed, see for example, Slob (2002). The dose-response model is fitted on the log-scale, where both the model and the data are log-transformed. After fitting the model, the model and the data may be backtransformed to the original scale for purposes of plotting and interpretation. We now show how our method can be extended to accommodate the log-normality assumption to find optimal designs.

It suffices to note here that we now assume logarithm of different observations are independent with the same variance, say $\sigma^{2}>0$, and have expectation

$$
E[\ln Y]=\ln \eta(t, \theta)
$$

where $\eta(t, \theta)$ is one of the 4 functions listed at the beginning of Section 2. Proceeding as in Section 2, one obtains an expression for the CED and the asymptotic variance of the estimated CED. The key difference is that the information matrix is now given by

$$
\tilde{M}(\xi, \theta)=\int_{0}^{T} \tilde{f}(t, \theta) \tilde{f}^{T}(t, \theta) d \xi(t)
$$

where $\tilde{f}(t, \theta)=\frac{1}{\eta(t, \theta)} \frac{\partial \eta(t, \theta)}{\partial \theta}$.

It follows that the vector $\tilde{f}(t, \theta)$ for each model is now different from the one under normality assumption in Section 2. However, the vector $g(\theta)$ for each model remains the same whether we assume the errors are normally or log-normally distributed. The next few tables display selected maximin CED-optimal designs (Table 6), maximin compound optimal designs (Table 8) under log-normal assumption and their efficiencies. From Tables 3 and 6 , we observe that the maximin optimal designs obtained under the normality and log-normality assumptions do not appear to be substantially different. The same is observed for maximin compound designs in Tables 4 and 7 .

\subsection{Efficiencies of commonly used designs}

Now we discuss efficiencies of designs described in Section 1 relative to our proposed optimal designs. Recall that these are some of the types of designs commonly used by toxicologists in practice. Specifically, designs and nominal values of parameters are taken from Woutersen et al. (2001) and Piersma et al. (2002). We list their various efficiencies 
Table 7. Maximin compound designs for models (2.3)-(2.6) with lognormality assumption on the design space $[0, T]$ for various values of the parameter $b$ with $d=1, c=0$ and $\mathrm{CES}=0.05$

\begin{tabular}{cccccccccc}
\hline$T$ & $b$ & $t_{1}$ & $t_{2}$ & $t_{3}$ & $t_{4}$ & $w_{1}$ & $w_{2}$ & $w_{3}$ & $w_{4}$ \\
\hline 1 & 0.1 & 0 & 0.176 & 0.628 & 1 & 0.374 & 0.155 & 0.271 & 0.200 \\
1 & 0.5 & 0 & 0.196 & 0.638 & 1 & 0.334 & 0.224 & 0.221 & 0.221 \\
1 & 1.0 & 0 & 0.199 & 0.659 & 1 & 0.325 & 0.202 & 0.242 & 0.232 \\
5 & 0.1 & 0 & 0.981 & 3.191 & 5 & 0.334 & 0.224 & 0.221 & 0.221 \\
5 & 0.5 & 0 & 1.174 & 3.469 & 5 & 0.322 & 0.199 & 0.241 & 0.238 \\
5 & 1.0 & 0 & 1.357 & 3.683 & 5 & 0.312 & 0.206 & 0.250 & 0.233 \\
\hline
\end{tabular}

in Table 9 relative to our optimal designs constructed under the log-normality assumption. Generally, the efficiencies of these designs are low for estimating CED or for discriminating between competing models in the stipulated class. They range from as low as $2 \%$ to mostly below $50 \%$, and in a couple of instances about $67 \%$ for model discrimination.
We see that in nearly all cases the minimal efficiency of the maximin compound design is greater than the efficiencies of the designs used by toxicologists. Additional calculations not shown here also reveal that the efficiencies of maximin compound designs are higher than the corresponding efficiencies of the commonly used designs by at least $10 \%$. This means that any confidence interval for CED constructed from the maximin optimal design is at least $10 \%$ shorter than those from the commonly used designs. In many cases the improvement is even more substantial. We also compare performance of these commonly used designs with optimal designs constructed under the normality assumption and the overall results are quite similar.

\subsection{Performance of maximin optimal design in practice}

All our optimal designs were found under a large sample assumption. These optimal designs minimize the asymptotic variance of the estimated CED obtained via the delta

Table 8. CED-Efficiencies and efficiencies of discrimination for compound optimal designs for models (2.3)-(2.6) on the design space $[0, T]$ with lognormality assumption and various values of the parameter $b$ with $d=1, c=0$ and $\mathrm{CES}=0.05$

\begin{tabular}{|c|c|c|c|c|c|c|c|c|c|}
\hline \multirow{3}{*}{$T$} & \multirow{3}{*}{$b$} & \multicolumn{4}{|c|}{ CED efficiency } & \multicolumn{4}{|c|}{ Efficiency of discrimination } \\
\hline & & $(2.3)$ & $(2.4)$ & $(2.5)$ & $(2.6)$ & $(2.4)-(2.3)$ & $(2.5)-(2.3)$ & $(2.6)-(2.5)$ & $(2.6)-(2.4)$ \\
\hline & & \multicolumn{8}{|c|}{ maximin compound design } \\
\hline 1 & 0.1 & 0.614 & 0.622 & 0.627 & 0.614 & 0.628 & 0.648 & 0.614 & 0.688 \\
\hline 1 & 0.5 & 0.620 & 0.655 & 0.620 & 0.620 & 0.675 & 0.628 & 0.721 & 0.727 \\
\hline 1 & 1.0 & 0.636 & 0.636 & 0.636 & 0.636 & 0.645 & 0.636 & 0.707 & 0.753 \\
\hline 5 & 0.1 & 0.620 & 0.655 & 0.620 & 0.620 & 0.675 & 0.628 & 0.722 & 0.727 \\
\hline 5 & 0.5 & 0.643 & 0.643 & 0.643 & 0.643 & 0.649 & 0.643 & 0.678 & 0.730 \\
\hline 5 & 1.0 & 0.640 & 0.640 & 0.640 & 0.640 & 0.644 & 0.640 & 0.662 & 0.729 \\
\hline
\end{tabular}

Table 9. CED-Efficiencies and efficiencies of discrimination for designs used by toxicologists for models (2.3)-(2.6) with lognormality assumption on the design space $[0, T]$ for various values of the parameter $b, d$ and $c$ and $\mathrm{CES}=0.05$ where $M^{*}$ is the minimal efficiency of maximin compound design

\begin{tabular}{|c|c|c|c|c|c|c|c|c|c|c|c|}
\hline \multirow{3}{*}{$b$} & \multirow{3}{*}{$c$} & \multirow{3}{*}{$d$} & \multirow{3}{*}{$M^{*}$} & \multicolumn{4}{|c|}{ CED efficiency } & \multicolumn{4}{|c|}{ Efficiency of discrimination } \\
\hline & & & & $(2.3)$ & $(2.4)$ & $(2.5)$ & $(2.6)$ & $(2.4)-(2.3)$ & $(2.5)-(2.3)$ & $(2.6)-(2.5)$ & $(2.6)-(2.4)$ \\
\hline & & & & \multicolumn{8}{|c|}{ design $0,0.5,1.5,3,4.5,6,7.5$ (in $100 \mathrm{mg} / \mathrm{kg}$ scale), $T=7.5$} \\
\hline 0.04 & 1 & 0 & 0.597 & 0.492 & 0.504 & 0.563 & 0.361 & 0.558 & 0.579 & 0.594 & 0.666 \\
\hline 0.06 & 1 & 0 & 0.616 & 0.492 & 0.517 & 0.570 & 0.419 & 0.558 & 0.579 & 0.594 & 0.666 \\
\hline 0.09 & 1 & 0 & 0.628 & 0.492 & 0.526 & 0.574 & 0.464 & 0.558 & 0.579 & 0.594 & 0.665 \\
\hline \multicolumn{12}{|c|}{ design $0,0.5,1.5,4.5$ (in $100 \mathrm{mg} / \mathrm{kg}$ scale), $T=7.5$} \\
\hline 0.04 & 1 & 0 & 0.597 & 0.217 & 0.375 & 0.185 & 0.551 & 0.245 & 0.057 & 0.126 & 0.032 \\
\hline 0.06 & 1 & 0 & 0.616 & 0.217 & 0.348 & 0.165 & 0.497 & 0.245 & 0.053 & 0.121 & 0.028 \\
\hline 0.09 & 1 & 0 & 0.628 & 0.217 & 0.331 & 0.150 & 0.375 & 0.245 & 0.047 & 0.114 & 0.024 \\
\hline \multicolumn{12}{|c|}{ design $0,0.5,1.5,4.5$ (in $100 \mathrm{mg} / \mathrm{kg}$ scale), $T=4.5$} \\
\hline 0.04 & 1 & 0 & 0.607 & 0.602 & 0.526 & 0.398 & 0.548 & 0.681 & 0.498 & 0.370 & 0.294 \\
\hline 0.06 & 1 & 0 & 0.596 & 0.602 & 0.566 & 0.406 & 0.517 & 0.681 & 0.493 & 0.364 & 0.286 \\
\hline 0.09 & 1 & 0 & 0.612 & 0.602 & 0.590 & 0.406 & 0.490 & 0.681 & 0.485 & 0.356 & 0.274 \\
\hline \multicolumn{12}{|c|}{ design $0,1.0,1.7,2.8,4.7,7.8,13,22,36,60, T=60$} \\
\hline 0.10 & 1 & 0 & 0.638 & 0.380 & 0.491 & 0.352 & 0.360 & 0.493 & 0.328 & 0.346 & 0.257 \\
\hline 0.10 & .5 & 0 & 0.613 & 0.380 & 0.440 & 0.352 & 0.478 & 0.488 & 0.328 & 0.613 & 0.640 \\
\hline 0.04 & .5 & 0 & 0.609 & 0.380 & 0.411 & 0.449 & 0.360 & 0.488 & 0.417 & 0.610 & 0.641 \\
\hline 0.10 & 1 & .9 & 0.614 & 0.380 & 0.491 & 0.417 & 0.319 & 0.493 & 0.529 & 0.551 & 0.265 \\
\hline
\end{tabular}


Table 10. Simulated normalized variance of CED for several true values of parameters

\begin{tabular}{ccccc}
\hline$b$ & $d$ & $c$ & $\operatorname{Var}(\widehat{C E D})$ with $\xi_{m m}$ & $\operatorname{Var}(\widehat{C E D})$ with $\xi_{u}$ \\
\hline 0.10 & 1.0 & 0.0 & 3.77 & 5.56 \\
0.10 & 0.5 & 0.0 & 172.1 & 250.3 \\
0.06 & 0.5 & 0.0 & 1821.0 & 3011.2 \\
0.10 & 1.0 & 0.2 & 13.84 & 20.81 \\
0.10 & 1.0 & 0.9 & 1850.4 & 3106.8 \\
\hline
\end{tabular}

method. For this reason it is important to investigate the superiority of the optimal designs for sample size observed in practice. The purpose here is to briefly compare variances of the estimated CED from the maximin CED-optimal design and a design used by toxicologists in a real example with a relatively small sample size.

In Piersma et al. (2002), rats were prenatally exposed to diethylstilbestrol and the implemented design $\xi_{u}$ had 6 animals in each of the 10 dose groups at $0,1.0,1.7,2.8$, $4.7,7.8,13,22,36$ and $60 \mathrm{mg} / \mathrm{kg}$ body weight per day. This means that we have 60 observations on the design space $[0,60]$. The maximin CED-optimal design $\xi_{m m}$ for $b=0.1, d=1, c=0$ has four dose levels and is given by $\{0,5.2,15.4,60 ; 14 / 60,21 / 60,19 / 60,6 / 60\}$.

We simulate data with $a=1$ and $\sigma=0.05$ and several values of parameters $b, d$ and $c$ with 1000 replicates in each simulation. In Table 10 we report simulated normalized variances of the estimated CED from the two designs under normality assumption. It is reassuring that we observe that in all the cases considered here, the variance obtained from the maximin optimal design $\xi_{m m}$ is consistently smaller than the variance obtained from the design $\xi_{u}$ used in practice. This implies that use of our proposed designs can save experimental cost for toxicologists and more importantly, in reducing the number of animals required in the study. In addition, unlike designs used by toxicologists, our designs are based on firm statistical considerations.

\subsection{A design web site for practitioners}

We conclude this paper with a reference to our web site where algorithms for generating optimal designs in this paper have been implemented. We believe that to facilitate use of optimal design ideas in practice, design tools should be readily available to practitioners. We thank the National Institute of General Medical Sciences for funding the construction and maintenance of the site. This site is housed at http://www.optimal-design.org/ and at http://optimal-design.biostat.ucla.edu/optimal/. Visitors to these sites can freely generate a variety of optimal designs for a broad range of models listed on the site. The site also contains introductory optimal design material.

Presently, the site contains a list of models commonly used in the biological sciences, along with information and references on optimal design issues. The visitor selects an appropriate model, an optimality criterion and inputs parameters for the design problem. The generated design is displayed, and when appropriate, is also accompanied by a plot of the directional derivative of the optimality criterion. Depending on the features exhibited in this plot, we may or may not confirm the optimality of the generated design over all designs on the design space. This site also calculates efficiencies of user-supplied designs so that practitioners can easily compare their designs with the optimum and make an informed decision whether to stray away from the optimum and if so by how much. The program for generating the proposed optimal designs in this paper can be found on our web site under the link "Optimal Designs for Toxicology Studies." For space considerations, we omit discussion and refer the reader to our web site for details. We hope that the site will promote use of optimal design ideas in practice.

\subsection{Closing remarks}

The optimal designs constructed in this paper are all locally optimal in the sense that nominal values of the model parameters are required to implement the designs. These nominal values are usually obtained from pilot studies or estimates from the literature for similar studies. Clearly inaccurate nominal values lead to a poor design, and so nominal values should be selected carefully. Locally optimal designs were proposed by Chernoff (1953) and they remain to date a useful first step in the construction of an efficient design for a nonlinear model.

In practice, the optimal design is constructed sequentially. One uses the current nominal values of the model parameters to construct the locally optimal design, and use the design to estimate the model parameters. Our experience is that after a few iterations, there is convergence and the optimal design becomes stable and does not change anymore with more iterations. An efficient way to achieve convergence is to use the sequential approach discussed in $\mathrm{Hu}$ and Zhang (2004) for finding an optimal design. Alternatively, a response-adaptive approach can also be applied to target the optimal design sequentially; see $\mathrm{Hu}$ and Rosenberger (2006) for details.

Received 5 December 2008

\section{REFERENCES}

[1] Biedermann S., Dette, H., and Pepelyshev, A. (2006). Some robust design strategies for percentile estimation in binary response models. Canad. J. Statist. 34 603-622. MR2347048

[2] Brown, L. D., Olkin, I., Sacks, J., and Wynn, H. P. (1985). Jack Carl Kiefer Collected Papers III: Design of experiments. Springer-Verlag, New York. MR0771481

[3] Catalano, P. J., Scharfstein, D. O., Ryan, L. M., KimMeL, C. A., and Kimmel, G. L. (1993). Statistical model for fetal death, fetal weight, and malformation in developmental toxicity studies. Teratology 47 281-290.

[4] Chernoff, H. (1953). Locally optimal designs for estimating parameters. Ann. Math. Statist. 24 586-602. MR0058932 
[5] Dette, H. (1995). Optimal designs for identifying the degree of a polynomial regression. Ann. Statist. 23 1248-1266. MR1353505

[6] Dette, H., Bretz, F., Pepelyshev, A., and Pinheiro, J. (2008). Optimal designs for dose finding studies. Journal of the American Statistician Association. In press. MR2462895

[7] Dette, H., Melas, V. B., and Wong, W. K. (2005). Optimal design for goodness-of-fit of the Michaelis-Menten enzyme kinetic function. Journal of the American Statistician Association 100 1370-1381. MR2236448

[8] Gaylor, D. W. and Chen, J. J. (1993). Dose-response models for developmental malformations. Teratology 47 291-297.

[9] Hu, F. and Rosenberger, W. F. (2006). The Theory of Response-Adaptive Randomization in Clinical Trials. Wiley, N.Y. MR2245329

[10] Hu, F. and Zhang, L. (2004). Asymptotic properties of doubly adaptive biased coin designs for multitreatment clinical trials. Annals of Statistics 32 268-301. MR2051008

[11] Moerbeek, M., Piersma, A. H., and Slob, W. (2004). A comparison of three methods for calculating confidence intervals for the benchmark dose. Risk Analysis 24 31-40.

[12] Müller, C. H. (1995). Maximin efficient designs for estimating non-linear aspects in linear models. J. Statist. Plann. Inference 44(1) 117-132. MR1323074

[13] Müller, C. H. and Pázman, A. (1998). Applications of necessary and sufficient conditions for maximin efficient designs. Metrika 48 1-19. MR1647889

[14] Oscar, T. (2004). Dose-response model for 13 strains of Salmonella. Risk Analysis 24 41-49.

[15] PÁzman, A. (1986). Foundations of optimum experimental design. D. Reidel Publishing Company. MR0838958

[16] Piersma, A. H., Verhoef, A., Sweep, C. G. J., De Jong, W. H., and van Loveren, H. (2002). Developmental toxicity but no immunotoxicity in the rat after prenatal exposure to diethylstilbestrol. Toxicology $\mathbf{1 7 4} 173-181$.

[17] Pukelsheim, F. (1993). Optimal design of experiments. Wiley, N.Y. MR1211416

[18] Rieder, S. and Pukelsheim, F. (1992). Efficient rounding of approximate designs. Biometrika 79 763-770. MR1209476

[19] Slob, W. (2002). Dose-response modeling of continuous end- points. Toxicological Sciences 66 298-312.

[20] Slob, W. and Pieters, M. N. (1998). A probabilistic approach for deriving acceptable human intake limits and human health risks from toxicological studies: general framework. Risk Analysis 18 787-798.

[21] Woutersen, R. A., Jonker, D., Stevenson, H., te BieseBEEK, J. D., and SLOB, W. (2001). The benchmark approach applied to a 28-day toxicity study with Rhodorsil Silane in rats: the impact of increasing the number of dose groups. Food and Chemical Toxicology 39 697-707.

\section{Holger Dette}

Ruhr-Universität Bochum

Fakultät für Mathematik

44780 Bochum, Germany

E-mail address: holger.dette@ruhr-uni-bochum.de

Andrey Pepelyshev

St. Petersburg State University

Department of Mathematics

St. Petersburg, Russia

E-mail address: andrey@ap7236.spb.edu

Piter Shpilev

St. Petersburg State University

Department of Mathematics

St. Petersburg, Russia

E-mail address: pitsh@front.ru

Weng Kee Wong

UCLA

Department of Biostatistics

Los Angeles, CA 90095, USA

E-mail address: wkwong@ucla.edu 